\title{
Thermoresponsive fluorescence switches based on Au@pNIPAM nanoparticles
}

Dana Kamzabek, ${ }^{a}$ Brieuc Le Dé, ${ }^{a}$ Liliane Coche-Guérente, ${ }^{b}$ Fabien Miomandre a and Galina V. Dubacheva ${ }^{a, b *}$

a PPSM, CNRS, Ecole Normale Supérieure Paris-Saclay, Université Paris-Saclay, 61 Avenue Président Wilson, 94235 Cachan, France. ${ }^{b}$ Department of Molecular Chemistry, Univ. Grenoble Alpes, CNRS UMR 5250, 570 rue de la chimie, CS 40700, Grenoble 38000, France.

\section{Supplementary Information}

1. UV-visible and fluorescence characterization of pNIPAM-FAM

2. AFM, fluorescence and DLS characterization of Au NP/pNIPAM-FAM 


\section{UV-visible and fluorescence characterization of pNIPAM-FAM}

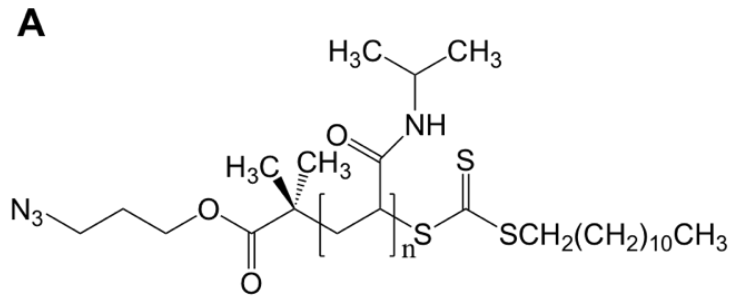

pNIPAM- $\mathrm{N}_{3}$

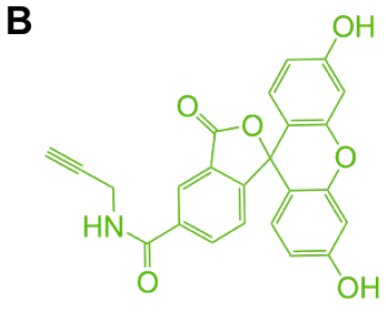

FAM-alkyne

Fig. S1. Chemical structures of (A) bifunctional pNIPAM derivative, bearing azide and protected thiol groups from the opposite sides (average $M_{n}=15000 \mathrm{~g} / \mathrm{mol}, P D I \leq 1.3$, SigmaAldrich) and (B) 5-carboxyfluorescein alkyne (FAM-alkyne, 5-isomer, 97\%, Lumiprobe).
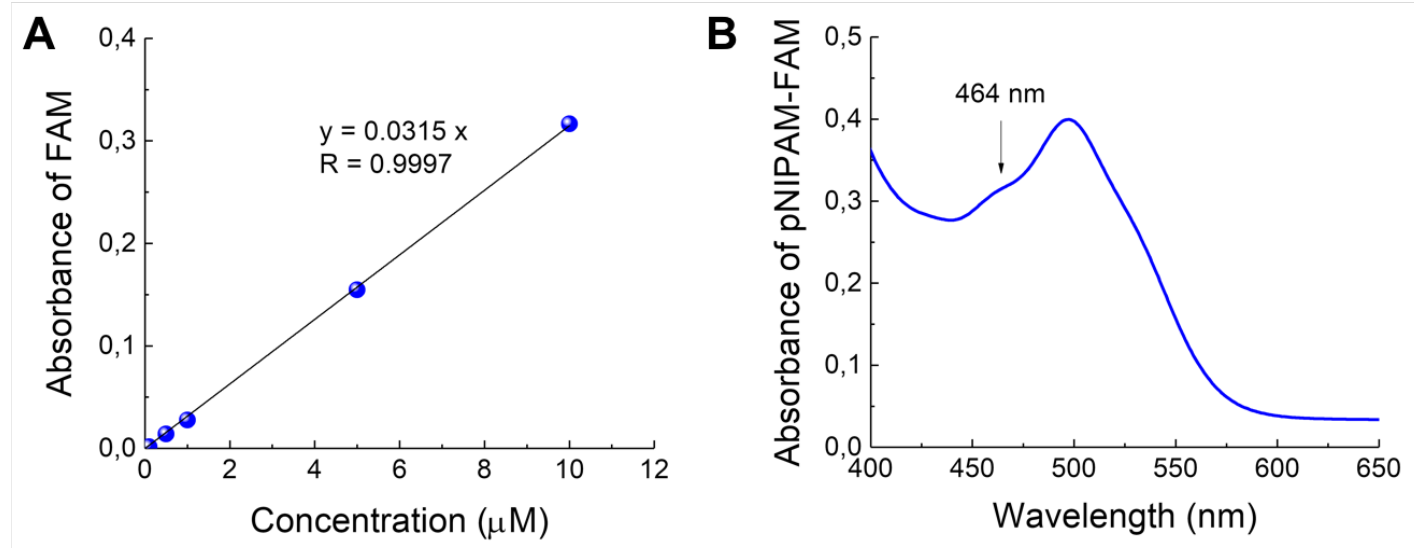

Fig. S2. UV-vis characterization of pNIPAM-FAM. (A) Absorbance of FAM-alkyne at 464 $n m$ recorded in $0.1 \mathrm{M}$ phosphate buffer ( $\mathrm{pH} 8$ ) and plotted versus FAM-alkyne concentration. (B) Absorbance spectrum of the $32 \mu M$ pNIPAM-FAM aqueous solution.

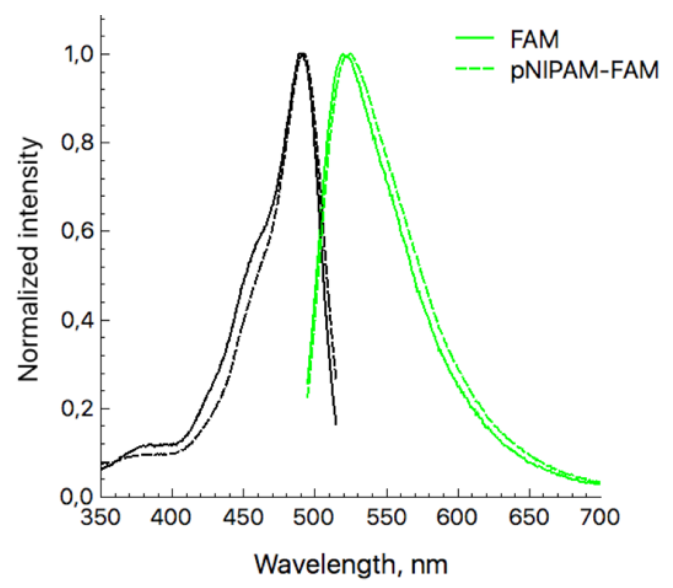

Fig. S3. Fluorescence characteristics of FAM and pNIPAM-FAM. Excitation (black) and emission (green) spectra of FAM (solid lines) and pNIPAM-FAM (dashed lines). Conditions: $20{ }^{\circ} \mathrm{C}$, samples prepared in water, emission spectra recorded while exciting at $480 \mathrm{~nm}$, excitation spectra recorded at emission wavelength fixed to $530 \mathrm{~nm}$. 


\section{AFM, fluorescence and DLS characterization of Au NP/pNIPAM-FAM}
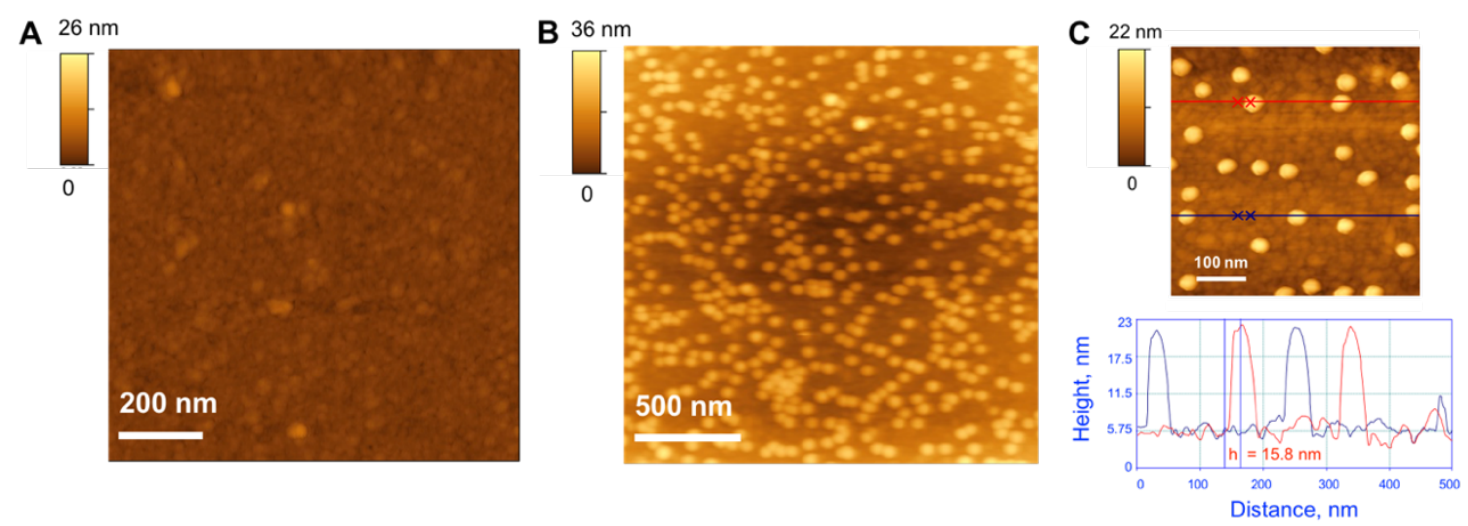

Fig. S4. AFM characterization of Au NPs. (A) Representative 2D topography image of the bare ITO surface (A), citrate-stabilized Au NPs attached to the ITO surface (B) and an example of the line analysis illustrating the heights of the nano-objects $(C)$.

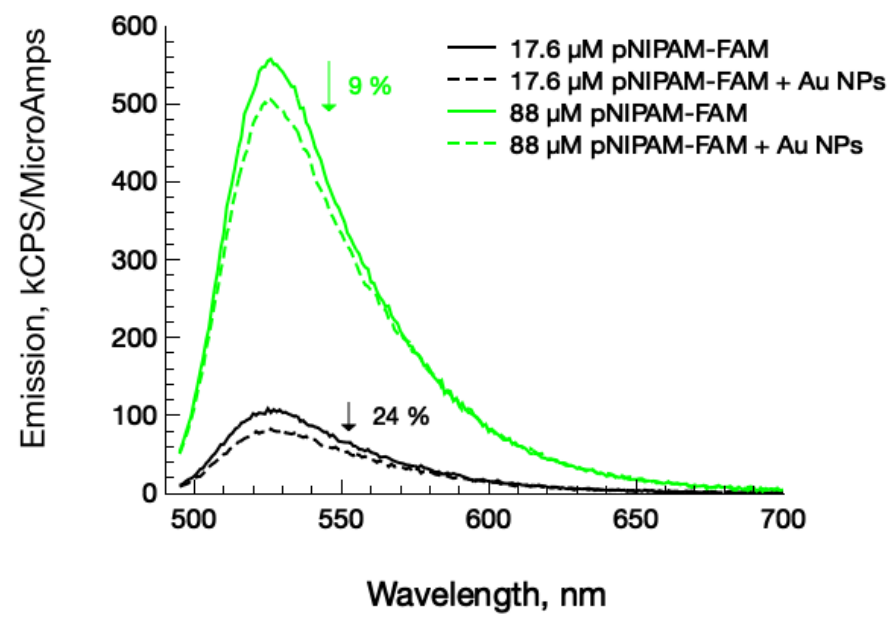

Fig. S5. Fluorescence characteristics of Au NP/pNIPAM-FAM at the excess of pNIPAMFAM. Emission spectra of pNIPAM-FAM obtained in the absence (solid lines) and in the presence (dashed lines) of Au NPs at 10 (black) and 50 (green) pNIPAM-FAM/nm ${ }^{2}$ ratios. The quenching efficiency $Q=\left(I_{\max }-I_{\max }^{A u N P}\right) \times 100 \% / I_{\max }$ is indicated in each case, with $I_{\max }$ and I $I_{\max }^{A u}$ being the maximal intensities of pNIPAM-FAM emission in the absence and in the presence of $A u$ NPs. Conditions: $20{ }^{\circ} \mathrm{C}$, citrate-stabilized $A u$ NPs $\left(c_{A u 0}=0.26 \mathrm{mM}\right)$ functionalized with pNIPAM-FAM ( $c_{P N I P A M-F A M}=17.6$ or $\left.88 \mu M\right)$, samples diluted 5 times with water, pNIPAM-FAM excitation at $480 \mathrm{~nm}$, pNIPAM-FAM emission at $530 \mathrm{~nm}$.

\section{Fluorescence characterization of dialyzed Au NP/pNIPAM-FAM}

Au NP/pNIPAM-FAM assemblies were exposed to dialysis and analyzed by fluorescence to estimate the fraction of unbound pNIPAM-FAM. Fig. S6A shows fluorescence emission spectra of the dialysis fluids ( $400 \mathrm{~mL}$ of $2 \mathrm{mM}$ citrate buffer at $\mathrm{pH}$ 6), to which pNIPAM-FAM and $\mathrm{Au} \mathrm{NP/pNIPAM-FAM} \mathrm{samples}(1 \mathrm{~mL}, 1.76 \mu \mathrm{M}$ pNIPAM-FAM corresponding to 1 pNIPAM-FAM $/ \mathrm{nm}^{2}$ ) were exposed inside the dialysis membranes with $\mathrm{MWCO}=50 \mathrm{~kg} / \mathrm{mol}$ (regenerated cellulose membrane tubing Spectra/Por 7). The comparison of the maximal emission intensities suggests that only $\sim 16 \%$ of pNIPAM-FAM was unbound in the $\mathrm{Au}$ $\mathrm{NP} / \mathrm{pNIPAM}-\mathrm{FAM}$ sample at $1 \mathrm{pNIPAM}-\mathrm{FAM} / \mathrm{nm}^{2}$ grafting density. The same experiment was performed at 10-times higher pNIPAM-FAM concentration (Fig. S6A inset). In this case, 
the obtained maximal emission intensities differ by only $\sim 21 \%$, which suggests that the majority of pNIPAM-FAM $(\sim 79 \%)$ was unbound in the case of 10 pNIPAM-FAM $/ \mathrm{nm}^{2}$ sample.

To maximize the stability of Au NPs, the dialysis was performed in $2 \mathrm{mM}$ citrate buffer at $\mathrm{pH} 6$ instead of water (Fig. S6B). The dialysis membrane with $\mathrm{MWCO}=50 \mathrm{~kg} / \mathrm{mol}$ was chosen as it was impenetrable for Au NPs (Fig. S6B), while allowing efficient release of free pNIPAMFAM into the dialysis fluid (Fig. S6C). Indeed, > $95 \%$ decrease in pNIPAM-FAM fluorescence emission was detected for 1.76 and $17.6 \mu \mathrm{M}$ pNIPAM-FAM samples ( $3 \mathrm{~mL}$ exposed to 400 $\mathrm{mL}$ of dialysis fluid); the tested concentrations correspond to 1 and $10 \mathrm{pNIPAM-FAM} / \mathrm{nm}^{2}$ ratios in the case of Au NP/pNIPAM-FAM assemblies (Fig. S6C).
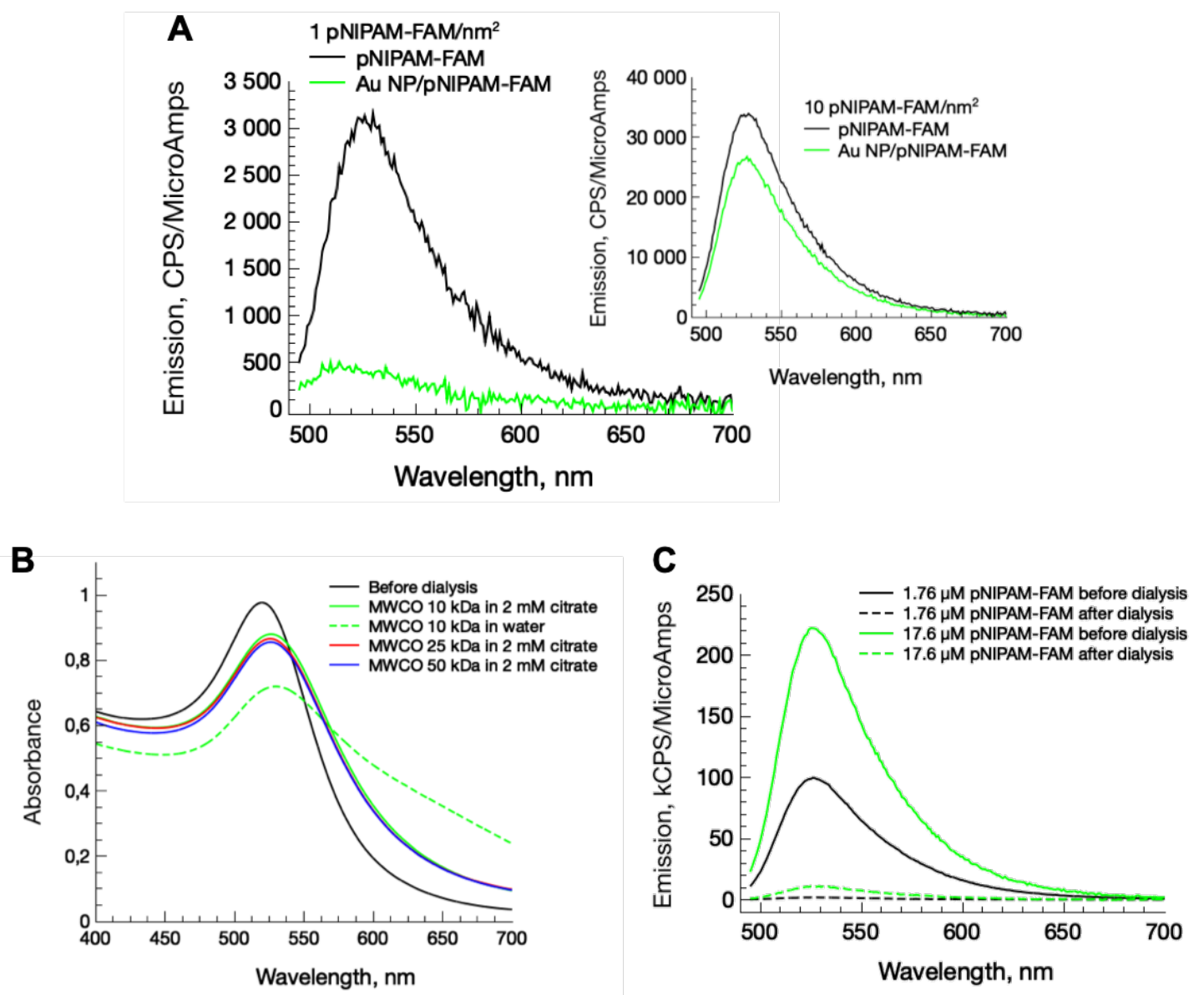

Fig. S6. Fluorescence and UV-visible characterization of dialyzed pNIPAM-FAM and Au NP/pNIPAM-FAM. (A) Emission spectra of dialysates (2 mM citrate buffer, pH 6) of pNIPAMFAM (black) and Au NP/pNIPAM-FAM (green) at 1 pNIPAM-FAM/nm ${ }^{2}$ ratio (c $c_{P N I P A M-F A M}$ $=1.76 \mu M)$. Inset: Emission spectra of dialysates (2 mM citrate buffer, pH 6) of pNIPAM-FAM (black) and Au NP/pNIPAM-FAM (green) at 10 pNIPAM-FAM/nm ${ }^{2}$ ratio $\left(c_{P N I P A M-F A M}=17.6\right.$ $\mu M)$. (B) UV-visible absorbance spectra of citrate-stabilized Au NPs before dialysis (black), after dialysis in water at $M W C O=10 \mathrm{~kg} / \mathrm{mol}$ (green dashed line) and after dialysis in $2 \mathrm{mM}$ citrate buffer ( $\mathrm{pH} \mathrm{6)}$ at $\mathrm{MWCO}=10$ (green solid line), 25 (red) and 50 (blue) $\mathrm{kg} / \mathrm{mol}$. (C) Emission spectra of $1.76 \mu M$ (black) and 17.6 $\mu M$ (green) pNIPAM-FAM before (solid lines) and after (dashed lines) dialysis in $2 \mathrm{mM}$ citrate buffer ( $\mathrm{pH}$ 6) at $M W C O=50 \mathrm{~kg} / \mathrm{mol}$. Conditions: $20^{\circ} \mathrm{C}$, citrate-stabilized Au NPs $\left(c_{A u 0}=0.26 \mathrm{mM}\right), c_{P N I P A M-F A M}=1.76$ or $17.6 \mu \mathrm{M}$, samples are analyzed without dilution $(A, B)$ or at 10-times dilution with water $(C)$, blank spectrum (water) was subtracted from the recorded emission spectra $(A, C), p N I P A M-F A M$ excitation at $480 \mathrm{~nm}$, pNIPAM-FAM emission at $530 \mathrm{~nm}$. 


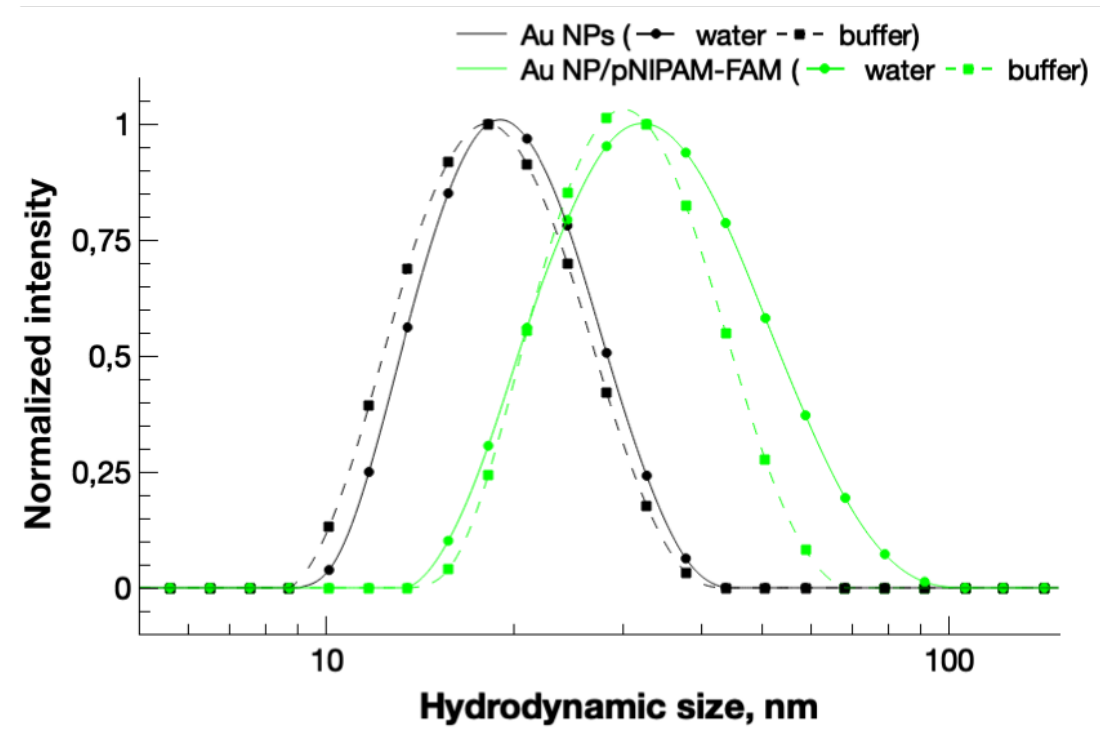

Fig. S7. DLS characterization of Au NPs and Au NP/pNIPAM-FAM in different media. Characteristic size distributions obtained by DLS before (black) and after (green) Au NPS incubation with $0.5 \mathrm{pNIPAM-FAM} / \mathrm{nm}^{2}$. The determined average sizes are summarized in the Table S1. Conditions: $20{ }^{\circ} \mathrm{C}$, citrate-stabilized Au NPs $\left(c_{A u 0}=0.26 \mathrm{mM}\right)$ functionalized with pNIPAM-FAM $\left(c_{P N I P A M-F A M}=0.9 \mu M\right)$, samples diluted 5- to 10-times with water (circles, solid lines) or $10 \mathrm{mM}$ sodium phosphate buffer at $\mathrm{pH} 8$ (squares, dashed lines).
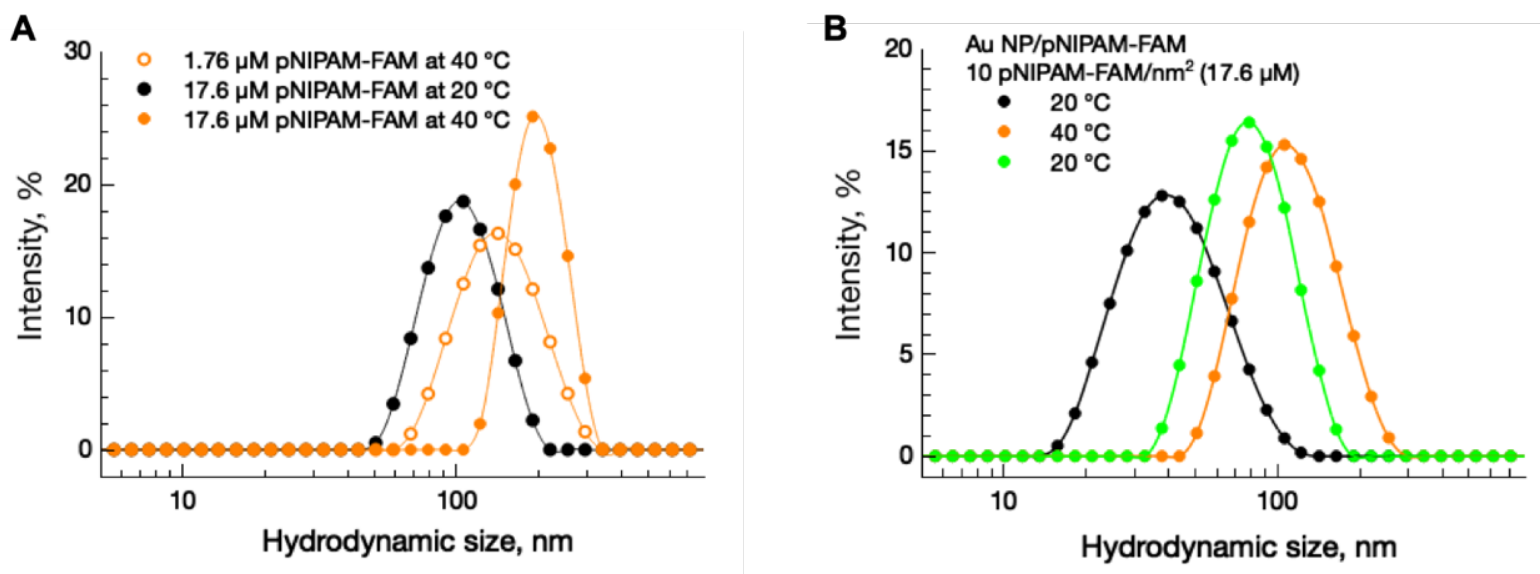

Fig. S8. DLS characterization of pNIPAM-FAM and Au NP/pNIPAM-FAM at the excess of pNIPAM-FAM. (A) Characteristic size distributions obtained for 1.76 (empty circles) and 17.6 $\mu M$ (filled circles) pNIPAM-FAM aqueous solutions at $20{ }^{\circ} \mathrm{C}$ (black) and $40{ }^{\circ} \mathrm{C}$ (orange). (B) Characteristic size distributions obtained for Au NP/pNIPAM-FAM assemblies at 10 pNIPAM$F A M / n^{2}$ ratio (corresponding to $17.6 \mu M$ pNIPAM-FAM) upon switching from $20{ }^{\circ} \mathrm{C}$ (black) to $40^{\circ} \mathrm{C}$ (orange) and back to $20^{\circ} \mathrm{C}$ (green). Conditions: temperature varied between 20 and $40{ }^{\circ} \mathrm{C}, c_{A u 0}=0.26 \mathrm{mM}, c_{P N I P A M-F A M}=1.76$ or $17.6 \mu \mathrm{M}$, samples diluted 5 times with water. 

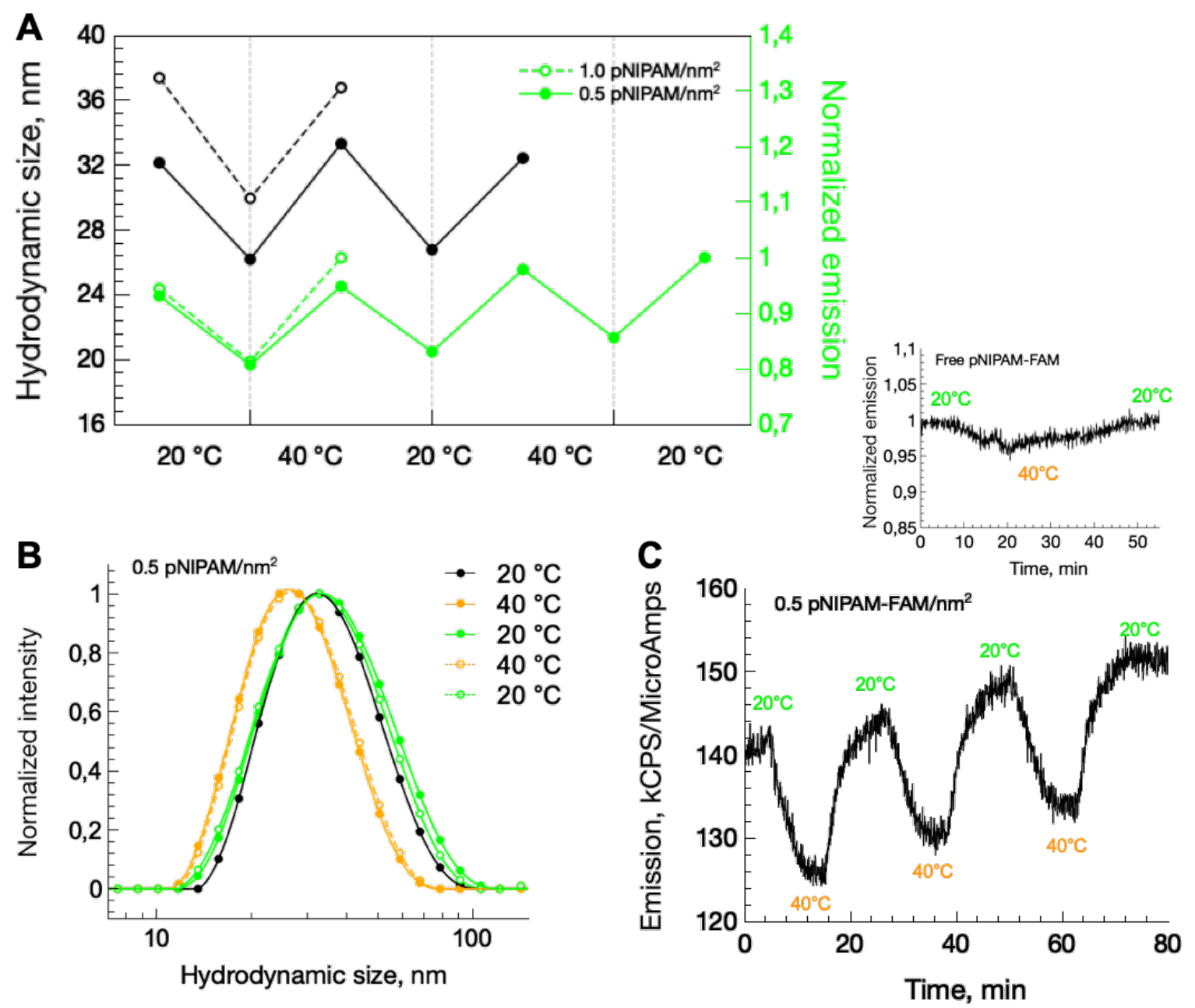

Fig. S9. Repeatability of Au NP/pNIPAM-FAM temperature response. (A) Changes in hydrodynamic size (left, black) and fluorescence emission (right, green) in response to switching between 20 and $40{ }^{\circ} \mathrm{C}$, registered for 0.5 (filled circles) and 1.0 (empty circles) pNIPAM-FAM $/ \mathrm{nm}^{2}$ ratios. The average hydrodynamic sizes are determined from size

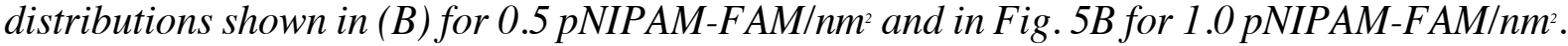
The fluorescence emission intensities are derived from kinetics measurements shown in $(C)$ for $0.5 \mathrm{pNIPAM-FAM} / \mathrm{nm}^{2}$ and in Fig. 5C for $1.0 \mathrm{pNIPAM-FAM} / \mathrm{nm}^{2}$. (B) Characteristic size distributions registered at $0.5 \mathrm{pNIPAM-FAM} / \mathrm{nm}^{2}$ grafting density in response to sequential 20/40/20/40/20 ${ }^{\circ} \mathrm{C}$ temperature switching. (C) Characteristic fluorescence kinetics at 0.5 pNIPAM-FAM/nm in response to sequential 20/40/20/40/20/40/20 ${ }^{\circ} \mathrm{C}$ temperature switching. Inset on top shows the temperature response of free PNIPAM-FAM recorded under the same

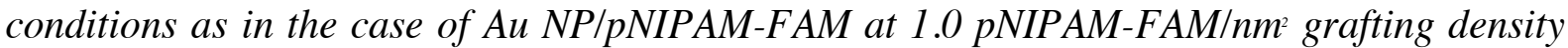
(Fig. 5C). Conditions: temperature varied between 20 and $40{ }^{\circ} \mathrm{C}$, citrate-stabilized Au NPs $\left(c_{\text {Aн }}=0.26 \mathrm{mM}\right)$ functionalized with pNIPAM-FAM $\left(c_{\text {PNPMM }}=0.88\right.$ or $\left.1.76 \mu \mathrm{M}\right)$, samples diluted 5-to 10-times with water (DLS measurements) or with $10 \mathrm{mM}$ sodium phosphate buffer, $\mathrm{pH}=$ 8 (fluorescence kinetics), pNIPAM-FAM excitation at $465 \mathrm{~nm}$, pNIPAM-FAM emission at 530 $n m$. The data shown in $(C)$ are obtained using Horiba Scientific Fluoromax-4 spectrofluorimeter equipped with a peltier cooler (LFI3751, Wavelength Electronics). 
Table S1. DLS results. Average hydrodynamic sizes $\left(D_{h}\right)$ determined at 20 or $40^{\circ} \mathrm{Cfor}$ different pNIPAM-FAM $/ \mathrm{nm}^{2}$ ratios based on the size distributions obtained by DLS. Examples of size distributions are shown in Figs. 2A, 5B, S7, S8B and S9B. Conditions: citrate-stabilized Au $N P s\left(c_{A u 0}=0.26 \mathrm{mM}\right)$ functionalized with pNIPAM-FAM $\left(c_{P N I P A M-F A M}=0.18-176.13 \mu \mathrm{M}\right)$, samples diluted 5-to 10-times with water or $10 \mathrm{mM}$ phosphate buffer $(\mathrm{pH}=8)$.

\begin{tabular}{|l|l|l|l|l|}
\hline $\begin{array}{l}\text { Grafting density, } \\
\text { pNIPAM- } \\
\text { FAM/nm }\end{array}$ & $\begin{array}{l}D_{h}^{20}{ }^{\circ} \mathrm{C} \text { before } \\
\text { heating to } 40 \\
\text { in water, } \mathrm{nm}\end{array}$ & $\begin{array}{l}D_{h}^{40}{ }^{\circ} \mathrm{C}, \\
\text { in water, } \\
\mathrm{nm}\end{array}$ & $\begin{array}{l}D_{h}^{20^{\circ} \mathrm{C}} \text { after } \\
\text { heating to } 40^{\circ} \mathrm{C}, \\
\text { in water, nm }\end{array}$ & $\begin{array}{l}D_{h}^{20}{ }^{\circ} \mathrm{C} \\
\text { in buffer, } \\
\text { nm }\end{array}$ \\
\hline $0^{\mathrm{a}}$ & $18.7 \pm 1.1$ & $18.8 \pm 1.1$ & $19.1 \pm 1.1$ & $17.8 \pm 1.4$ \\
\hline $0.1^{\mathrm{a}}$ & $22.4 \pm 1.3$ & - & - & - \\
\hline $0.2^{\mathrm{a}}$ & $27.2 \pm 1.6$ & $24.8 \pm 1.5$ & $26.6 \pm 1.6$ & - \\
\hline $0.5^{\mathrm{a}}$ & $31.7 \pm 1.9$ & $26.6 \pm 1.6$ & $30.9 \pm 1.9$ & $29.4 \pm 2.4$ \\
\hline $1.0^{\mathrm{a}}$ & $38.3 \pm 2.2$ & $31.0 \pm 1.9$ & $36.7 \pm 2.2$ & - \\
\hline $10.0^{\mathrm{a}}$ & $39.8 \pm 2.4$ & $107.1 \pm 6.4^{\mathrm{c}}$ & $77.8 \pm 4.7^{\mathrm{c}}$ & - \\
\hline $50.0^{\mathrm{b}}$ & $44.0 \pm 2.6$ & - & - & - \\
\hline $100.0^{\mathrm{b}}$ & $43.8 \pm 2.6$ & - & - & - \\
\hline
\end{tabular}

${ }^{a}$ Samples were characterized as prepared. ${ }^{\mathrm{b}}$ Samples were purified before DLS measurements as described elsewhere ${ }^{1}$ to remove the excess of free PNIPAM-FAM and thus minimize its contribution to the response at 20 ${ }^{\circ}{ }^{\circ} .{ }^{c}$ Strong changes in size indicate partially reversible aggregation resulting from enhanced inter-polymer interactions (Fig. S8B). 


\section{SPR characterization of PNIPAM-FAM surface density}

SPR experiments on planar gold sensors were performed to estimate the number of pNIPAM-FAM per surface area. SPR measurements and data analysis were performed as described previously. ${ }^{2,3}$ Briefly, SPR gold sensor chips (GE Healthcare) were cleaned by UV-ozone (15 min), immersed in ethanol $(15 \mathrm{~min})$ and dried in a nitrogen flow. The cleaned gold surface was dipped overnight in the $10 \mu \mathrm{M}$ pNIPAM-FAM aqueous solution. The surface was carefully cleaned with water and dried with nitrogen. The functionalized gold sensor chip was then mounted inside the Biacore T200 instrument (GE Healthcare) the the SPR signal was recorded in a water flow at $20^{\circ} \mathrm{C}$. The pNIPAM-FAM surface density was determined using the Jung's equation: ${ }^{4}$

$$
\Gamma=\Delta R U \frac{d}{\left.d n / d c^{\left(1-e^{-\frac{d}{d p}}\right.}\right)}
$$

where $\Gamma$ is the surface density of pNIPAM-FAM $\left(\mathrm{mol} / \mathrm{cm}^{2}\right), \Delta R U$ is the experimental SPR signal difference due to pNIPAM-FAM adsorption (resonance units $\times 10^{-6}$ ), $d$ is the thickness of pNIPAM-FAM layer $(\mathrm{cm}), d n / d c$ is the molar refractive index increment of pNIPAM-FAM $\left(\mathrm{cm}^{3} / \mathrm{mol}\right)$ and $d_{p}$ is the effective penetration depth of the evanescent wave $(175 \mathrm{~nm}) . \Delta R U$ was determined as:

$$
\triangle R U=R U_{A u / P N I P A M-F A M}-R U_{A u}
$$

where $R U_{A u}$ and $R U_{A u / p N I P A M-F A M}$ are the measured SPR signals of cleaned gold surface before and after its functionalization with pNIPAM-FAM (Table S2).

Table 2. SPR results. Mean resonance units values \pm standard errors obtained by averaging SPR signals measured in 4 microfluidic channels passing through different areas of the gold surface before and after its functionalization with pNIPAM-FAM.

\begin{tabular}{|l|l|}
\hline$R U_{A u}$ & $25797 \pm 250$ \\
\hline$R U_{A u / p N I P A M-F A M}$ & $33672 \pm 737$ \\
\hline$\Delta R U=R U_{A u / p N I P A M-F A M}-R U_{A u}$ & $7875 \pm 236$ \\
\hline
\end{tabular}

Assuming $d$ to be in the range from 6 to $13 \mathrm{~nm}$ (from $R_{\mathrm{g}}{ }^{5}$ to the maximal pNIPAM-FAM thickness determined by DLS, Fig. 3) and $d n / d c$ to be in the range from 0.133 to $0.167 \mathrm{~cm}^{3} / \mathrm{g}$ (according to the measured values reported in literature ${ }^{6-8}$ ), we obtained $\Gamma=64 \pm 8 \mathrm{pmol} / \mathrm{cm}^{2}$, which corresponds to $0.39 \pm 0.04 \mathrm{pNIPAM}-\mathrm{FAM} / \mathrm{nm}^{2}$. We notice that in the case of Au NPs, $\Gamma$ can be higher due to the longer incubation time, stirring during the assembly, absence of rinsing step and additional space available for pNIPAM-FAM insertion due to the nanoparticle curvature. ${ }^{9}$ 


\section{Supplementary references}

1. Jones, S. T. et al. The Importance of Excess Poly(N-isopropylacrylamide) for the Aggregation of Poly(N-isopropylacrylamide)-Coated Gold Nanoparticles. ACS Nano 10, 3158 3165 (2016).

2. Dejeu, J. et al. Impact of Conformational Transitions on SPR Signals-Theoretical Treatment and Application in Small Analytes/Aptamer Recognition. J. Phys. Chem. C 122, 21521-21530 (2018).

3. Prado, E. et al. Influence of the SPR Experimental Conditions on the G-Quadruplex DNA Recognition by Porphyrin Derivatives. Langmuir 34, 13057-13064 (2018).

4. Jung, L. S., Campbell, C. T., Chinowsky, T. M., Mar, M. N. \& Yee, S. S. Quantitative Interpretation of the Response of Surface Plasmon Resonance Sensors to Adsorbed Films. Langmuir 14, 5636-5648 (1998).

5. Jalili, K., Abbasi, F. \& Milchev, A. Surface Microdynamics Phase Transition and Internal Structure of High-Density, Ultrathin PHEMA-b-PNIPAM Diblock Copolymer Brushes on Silicone Rubber. Macromolecules 46, 5260-5278 (2013).

6. Kubota, K., Fujishige, S. \& Ando, I. Solution Properties of Poly( N isopropylacrylamide) in Water. Polym. J. 22, 15-20 (1990).

7. Zhou, S., Fan, S., Au-yeung, S. C. F. \& Wu, C. Light-scattering studies of poly(Nisopropylacrylamide) in tetrahydrofuran and aqueous solution. Polymer 36, 1341-1346 (1995).

8. Bongiovanni Abel, S., Riberi, K., Rivarola, C. R., Molina, M. \& Barbero, C. A. Synthesis of a Smart Conductive Block Copolymer Responsive to Heat and Near Infrared Light. Polymers 11, 1744 (2019).

9. Gibson, M. I. \& O'Reilly, R. K. To aggregate, or not to aggregate? considerations in the design and application of polymeric thermally-responsive nanoparticles. Chem. Soc. Rev. 42 , 7204-7213 (2013). 